\title{
TTR
}

Traduction, terminologie, re?daction

\section{Autour de la traduction inédite en français des Élégies roumaines d'Eugène Ionesco : texte et contexte de traduction pour appréhender le théâtre}

\section{Aurélia Roman}

Volume 3, numéro 1, 1er semestre 1990

L’agora de la traduction

URI : https://id.erudit.org/iderudit/037059ar

DOI : https://doi.org/10.7202/037059ar

Aller au sommaire du numéro

Éditeur(s)

Association canadienne de traductologie

ISSN

0835-8443 (imprimé)

1708-2188 (numérique)

Découvrir la revue

Citer cet article

Roman, A. (1990). Autour de la traduction inédite en français des Élégies

roumaines d'Eugène Ionesco : texte et contexte de traduction pour

appréhender le théâtre. TTR, 3(1), 63-82. https://doi.org/10.7202/037059ar

Tous droits réservés (C) TTR: traduction, terminologie, rédaction — Les auteurs, d'utilisation que vous pouvez consulter en ligne. 


\title{
Autour de la traduction inédite en français des Élégies roumaines d'Eugène Ionesco: texte et contexte de traduction pour appréhender le théâtre
}

Aurélia Roman

\begin{abstract}
Une cuvre d'homme n'est rien d'autre que ce long cheminement pour retrouver par les détours de l'art les deux ou trois images simples et grandes sur lesquelles le cour, une première fois, s'est ouvert.
\end{abstract}

Albert Camus

Si le théâtre d'Eugène Ionesco a fait l'objet d'innombrables études depuis le milieu de notre siècle, ses débuts littéraires en Roumanie, en tant que poète roumain, restent encore à explorer. Traductrice en français de ses premiers poèmes, nous voulons tout d'abord présenter au lecteur ne connaissant pas le roumain ces pièces poétiques encore inédites en français. On verra combien ces "œuvres de jeunesse» sont significatives en elles-mêmes et pour la compréhension juste du théâtre de l'auteur. Car tel est le deuxième objectif que nous visons: placer le théâtre de Ionesco dans la lumière ô combien éclairante, selon nous, des Élégies. Or, pour ce faire, une dimension doit être abordée sans détours, même si à ce jour elle n'a guère retenu l'attention des commentateurs, la dimension «bi-culturelle» ou, pour être plus précis, la dimension de traduction. Des constatations s'imposent en effet lorsqu'on cherche à saisir l'œuvre de Ionesco dans toute sa signifiance. Cette cuvre (et par là il faut bien sûr entendre en tout premier lieu le théâtre) trouve ses assises dans l'expérience bi-culturelle de l'au- 
teur. Sans entrer dans le détail de sa biographie, nous tenterons de dégager le contexte roumain dans lequel Ionesco évolue à l'époque de ses premières œuvres. Le fait que Ionesco (comme Beckett, cet autre géant de la dramaturgie du $20^{\mathrm{e}}$ siècle) vive une situation de biculturalisme nous paraît informer en profondeur son œuvre entier. Nous nous attacherons ici à mettre en lumière le versant roumain de l'œuvre et notamment celui du jeune poète des Élégies.

\section{Le contexte socio-culturel roumain}

Vingt ans avant la première de la Cantatrice chauve ${ }^{1}$, le futur fondateur du théâtre de l'absurde, du dérisoire et de l'avant-garde, entre en littérature par la poésie lyrique, genre qui semble tout à fait oublié et qui paraît accidentel dans son cas. Il s'agit d'une collection de brèves élégies intitulée Elegii pentru fiinte mici (Élégies pour petits êtres). Elles furent publiées pour la première fois entre les années 1928 et 1930 dans une revue quotidienne de Bucarest, Bilete de papagal (Billets de perroquet), revue dirigée par le plus grand poète roumain de l'époque, Tudor Arghezi. Le jeune Ionesco, qui en 1928 n'avait que dix-neuf ans, publia ses poèmes dans un petit volume, d'abord en 1931 à Craîova, ville roumaine entre les Carpathes et le Danube, près de son lieu de naissance, et ensuite en 1932 à Bucarest, en réédition.

C'est cette première cuvre d'Eugène Ionesco, à l'époque Eugen Ionescu, qui vient d'être traduite par nous et qui sera publiée prochainement avec les dessins de l'auteur. ${ }^{2}$

Les deux autres principaux ouvrages roumains de Ionesco, publiés peu de temps après les Élégies ont déjà été traduits au cours des dernières années. Ce sont Hugoliade: la vie grotesque et tragique de Victor Hugo (Gallimard, 1982), anti-biographie, pastiche du genre épique, et le volume d'essais critiques et de fragments de journal intime intitulé Non (Gallimard, 1986).

1. C'est-à-dire en 1932, puisque la première date de 1952. La Cantatrice chauve, première pièce de Ionesco, accomplit pour notre siècle (comme on l'a remarqué) ce qu'Hernani de Victor Hugo avait réussi pour le $\mathrm{XIX}^{\mathrm{t}}$, c'est-à-dire un renouvellement profond du thèâtre. Elle enregistre, $d$ 'ailleurs, un phénomène extraordinaire, unique dans l'histoire mondiale du théâtre, car depuis le 16 février 1957 et encore aujourd'hui cette pièce est jouée chaque soir au petit théâtre de la Huchette à Paris. Elle a battu le record mondial auparavant détenu par la Sorcière d'Agatha Christie, pièce qui fut jouée pendant 22 ans à Londres.

2. «En ce qui concerne la publication des Élégies, mes premiers et derniers poèmes, j'accepte qu'ils soient publiés uniquement pour vous... Je vais choisir quelques dessins pour illustrer ces poèmes: je vous les enverrai.» Eugène Ionesco, Lettre à Aurelia Roman du 9 Mars 1989. 
Si les poèmes de Ionesco ne sont pas encore traduits et connus, c'est surtout parce que l'auteur est gêné par ces «pièces que $\mathrm{j}$ 'ai faites quand $\mathrm{j}$ 'étais très jeune». ${ }^{3}$ Cette gêne est sans doute causée par leurs «maladresses» juvéniles, par l'incursion dans un genre où l'auteur ne trouva pas sa voie; elle peut aussi être due au fait que ces poèmes dévoilent une âme encore naïve et d'un sentimentalisme touchant. Ionesco a maintes fois insisté pour que l'on oublie ses «premiers et derniers poèmes» qu'il n'aime pas et qu'il rejette comme un mélange sentimental et symboliste "d'un anthropomorphisme rudimentaire", écrits sous l'influence de Maeterlinck et de Francis Jammes. Il est en outre fort probable que la première impulsion de jeunesse de la part du futur écrivain se soit exprimée, cela n'est pas inhabituel, par la poésie, forme qui correspond non seulement à la conviction populaire que «tout Roumain est né poète» ${ }^{4}$, mais encore au genre le plus pratiqué par ses contemporains en Roumanie. D'ailleurs, une lecture attentive de ses essais critiques de Non fournit certains éléments expliquant qu'il se soit laissé glisser sur la pente de la poésie et du lyrisme, lyrisme qui joue le rôle de contrepoids à ses écrits critiques agressifs et virulents de la même époque. Nous avons montré ailleurs (Théorie et pratique du monologue dans l'ceuvre de Ionesco, 1985, thèse non publiée) que le monologue remplira une fonction analogue dans ses pièces ultérieures, cuvre basée sur une multiple architecture de contraires.

En dépit de toutes les craintes et résérves de l'auteur vis-à-vis de ces poèmes de jeunesse, nous considérons que ces «pièces juvéniles» sont d'une incontestable importance pour l'exégèse de l'œuvre. 5

3. Lettre qu'Eugène lonesco nous adressait le 17 novembre 1989.

4. Létude d'Hubert Juin intitulée Introduction à la poésie roumaine (Paris, collection Messidor, 1961) en est un témoignage éloquent.

5. Pour célébrer les quatre-vingts ans $d^{\prime}$ Eugène Ionesco, $j$ 'ai eu l'idée de choisir les huit poèmes que j'aimais le plus du volume des Élégies pour petits êtres et de demander à notre ami le grand pianiste et compositeur Alan Mandel de les mettre en musique. Nous avons donc présenté la première mondiale de cette auvre inconnue le 6 novembre 1989 à l'Ambassade de France à Washington, D.C. Le spectacle eut un grand succès et fut acclamé par le critique d'art du Washington Post comme «one of the year's more unusual performing art events». Ce critique, Joseph McLellan, écrivit alors à propos des Élégies de Ionesco: «The early poems, written in Romanian and long forgotten, are no longer of interest to Ionesco himself, who long ago repudiated their late Romantic style. But they are expertly written and foreshadow some of his favorite themes as well as his mastery of words, ambiguities, absurdities and striking images, and in Roman's French translation they could easily hold their own with any poetry written today." (The Washington Post, November 8, 1989, p. C12). 
En premier lieu, les ignorer serait mal comprendre l'œuvre de l'auteur et méconnaître à quel point sa dramaturgie suit le chemin entrevu par le jeune poète. Pour Ionesco, la poésie «est cri et non discours» (Hugoliade, p. 26). Surprendre le jaillissement du cri, les images sous leur première forme, naïve ou gauche, «images sur lesquelles le cour une première fois s'est ouvert» et les retrouver sous tant d'autres aspects sur le chemin de la maturation de l'œuvre, nous semble une enquête passionnante et nécessaire. Mais il y a beaucoup plus. Limagination créatrice s'y révèle avec force, car on peut déjà identifier les thèmes obsessionnels et les structures qui caractériseront toute sa dramaturgie future. Cette constatation capitale pour l'exégèse de l'œuvre ionescienne infirme la position de certains critiques (voir justement les débats engagés par Ionesco avec la critique anglaise dans son célèbre volume d'essais critiques Notes et contre-notes, Gallimard, 1962) qui accusaient l'auteur d'avoir changé d'orientation et de «manière». L'œuvre entier de Ionesco, poésies, essais critiques, journaux intimes, contes, nouvelles et ses nombreuses pièces de théâtre, nous apparaît comme un tout organique dont les constantes soṇt identifiables dès ces premières pages publiées. Grâce à ces poèmes, nous sommes en mesure de mieux saisir, comprendre et apprécier la dramaturgie dans ce qu'elle aura d'essentiel et de plus original.

En second lieu, étant donné que l'œuvre roumaine a précédé le théâtre écrit en français, peut-être pourrons-nous mieux cerner ce que la spécificité de la dramaturgie ionescienne doit à l'expérience roumaine de l'auteur. La question sera donc aussi: dans quelle mesure y a-t-il une continuité, un héritage de cette expérience et des liens avec la production littéraire roumaine des années trente?

D'emblée, nous découvrons le fait essentiel que les ouvrages roumains de Ionesco s'affirment dans le cadre d'un courant littéraire négativiste d'une jeunesse en révolte. ${ }^{6}$ Cette littérature d'une génération agitée, tourmentée par la recherche exaspérée de son identité, vient après une époque appelée parfois amurgul zeilor (le crépuscule des dieux), c'est-à-dire après la mort des grands classiques de la littérature roumaine, qui écrivirent pendant la deuxième partie du XIX $\mathrm{XI}^{\mathrm{e}}$ siècle: le grand critique, polémiste et théoricien de l'esthétique littéraire Titu Maïoresco, le poète national Mihai Eminesco, l'incomparable conteur Ion Creangà, le grand

6. Emil Cioran publie, par exemple, Sur les cimes du désespoir et plus tard son célèbre Précis de décomposition. D. Rosca publie l'Existence tragique et Mihail Ilovici le Négativisme de la jeune génération, oủ il «essaie de définir ce courant négativiste». Voir E. Simion, Préface à Non, p. 19. 
dramaturge Ion Luca Caragiale. Ionesco n'hésite pas à reconnaître le rôle capital de ce dernier dans l'orientation de sa propre œuvre. Chez Caragiale, ce Molière des Roumains, dont Ionesco affirme qu'il «est probablement le plus grand des auteurs dramatiques inconnus», la caricature ubuesque de la bourgeoisie apparaît bien avant Jarry. Il en est de même pour l'absurde profond et amer qui s'exprime, selon Ionesco, par «les pires non-sens" s'accumulant «avec une richesse inépuisable». ${ }^{7}$ Les héros anti-héros de Caragiale sont les parents des Smith et des Martin, héros de la Cantatrice chauve, tandis que l'humour du langage fou qui se donnera libre cours dans les premières pièces de Ionesco y est déjà annoncé.

La période de l'entre-deux-guerres pendant laquelle Ionesco s'affirme en tant que poète et critique est une «période d'activité culturelle intense, d'effervescence d'idées et de courants». (E. Simion, préface de Non, p. 11) Ce bouillonnement est le résultat de l'enthousiasme et de l'essor qui caractérisent la «Grande Roumanie». Sont enfin réunies les provinces roumaines dont les principales sont la Valachie, la Moldavie et la Transylvanie. Toutes les villes roumaines voient fleurir une vie culturelle sans précédent, peut-être la plus intense et la plus libre jamais connue dans ce pays. Les revues prolifèrent, et le jeune Ionesco lui aussi publie des articles de critique littéraire dans une vingtaine d'entre elles, d'orientations très diverses. «La prose et la poésie connaissent un essor identique, tandis que se dessinent quelques grands courants d'idées.) (Non, p. 11) Certains s'appliquent à se débarrasser du «lyrisme paysan et idyllique» traditionnel et à «intellectualiser la poésie et citadiniser la prose», tandis que d'autres poursuivent le contraire en défendant une «littérature spécifiquement nationale»; les uns sont pour le symbolisme (certains sont des mallarméens de talent), les autres contre; enfin, il y a les promoteurs du réalisme historique et les partisans d'une approche purement esthétique ou d'une «avant-garde au programme constructiviste». Parmi ceux qui s'affirment ainsi on compte des écrivains, des poètes et des artistes qui seront également connus à l'étranger. Tristan Tzara s'était déjà fait connaître parmi les fondateurs du dadaïsme. Ajou-

7. Ionesco a fait un élogieux Portrait de Caragiale, dont une des pièces, «la Lettre perdue», a paru dans la collection les Hommes célebres: «I.L. Caragiale (18521912) est probablement le plus grand des auteurs dramatiques inconnus. Dégoûté par la société de son temps, ayant aggravé son dégoût en la dénigrant, dans toute son cuvre, avec violence et raison, talent et humour... Les héros de Caragiale sont fons de politique. Ce sont des crétins politiciens. $\hat{A}$ tel point qu'ils ont dèformé leur langage le plus quotidien... On signe avec courage une délation anonyme... On fait partie de tous les régimes parce qu'on est impartial.m (Notes et contre-notes, p. 117). 
tons Marcel Ianco, B. Fundoianu (Benjamin Fondane), Victor Brauner, Constantin Brâncusi, Ion Vinea, Ilarie Voronca, et de nombreux autres. Ces jeunes sont profondément marqués par leurs professeurs à l'université, tel le professeur d'esthétique Mihaï Dragomiresco auquel Ionesco se réfère à maintes reprises dans ses journaux, professeur qui «eut le mérite, dès 1893, avant Proust, de dissocier «la personnalité humaine» de la «personnalité créatrice», c'est-à-dire de distinguer le «moi social» du «moi profond»». (Non, p. 14) Autour du professeur de métaphysique Nae Ionesco (aucun lien de parenté avec notre auteur) se constitue le groupe Criterion auquel se rallient Mircea Eliade, futur historien des religions, Emil Cioran, de nos jours l'un des grands moralistes et philosophes d'expression française, les philosophes Constantin Noïca et Mircea Vulcanesco et plus tard Eugen Ionesco lui-même. Ils déploient tous une intense activité tant comme collaborateurs à de nombreuses revues que comme auteurs de leurs premières cuvres, dont une grande partie sont des poésies et des journaux intimes. Voilà donc, esquissé à larges traits, le monde effervescent et riche de controverses où voit le jour l'œuvre roumaine de Ionesco.

L'ouvre en prose, Hugoliade et Non, œuvre négativiste, scandalisa les lecteurs roumains des années trente. Le jeune auteur $s$ 'amusait à ridiculiser cruellement non seulement le grand poète Victor Hugo, mais les grands écrivains roumains de son temps. Cela ne l'empêche pas de remporter le prix des jeunes écrivains pour ce dernier volume. On y trouve déjà la formule originale de la dramaturgie, formule de l'absurde bien connue des Roumains: Non $=$ Oui $=$ Rien $=$ Rire ${ }^{8}$ Les résultats qu'elle produit sont assurés: surprise, choc, dérision, gêne, dissolution dans l'absurde et finalement rire. Il tourne en dérision les figures littéraires roumaines les plus importantes de l'époque, inaptes à faire œuvre valable, et il est prêt en même temps à soutenir le contraire, tout en gardant les mêmes critères! Ainsi, comme le remarque I. Gregori, «en guise de démonstration, il se livre à un exercice, désormais célèbre, en écrivant sur le roman de Mircea Éliade Maitreyi (La Nuit bengali) une première critique» où il "porte l'ouvrage aux nues, puis une seconde» où il «l'exécute». Ionesco fait savoir au lecteur que «Tout livre est remarquable si l'on se dit qu'il est

8. Nous avons découvert cette formule et démontré son fonctionnement dans notre thèse de doctorat sur Ionesco. 
remarquable. Tout livre est exécrable si l'on se dit qu'il est exécrable». (Non, pp. 302-303).

Enfin, pour conclure ces brèves remarques sur le contexte de l'œuvre roumaine de Ionesco, citons ces commentaires rétrospectifs de l'auteur qui nous paraissent fort justes:

Ces pages, qui datent d'un peu plus d'un demi-siècle, étaient l'cuvre d'un adolescent en colère... Mais à côté des maladresses et de quelques incohérences, ce qui a été dit alors, dans des affirmations plus profondes, plus spirituelles, j'ai continué à le dire et l'écrire tout au cours de ma vie et je le dirais encore aujourd'hui. (Non, Avant-propos)

Il nous apparaît donc essentiel, pour saisir Ionesco, de le replacer dans le contexte peu connu dans lequel les poèmes ont été écrits. L'œuvre ionescien entier doit aussi être considéré en tant que produit d'une époque où les jeunes écrivains tentent plus que jamais, et dans un effort très fécond, de réunir deux orientations opposées: s'assimiler aux grands courants de la littérature étrangère en général, et française en particulier, et travailler au développement d'une littérature fondée sur les sources nationales. Nous ne croyons pas possible d'apprécier pleinement l'cuvre de Ionesco sans la voir comme produit du biculturalisme, lieu de confluence et de cohésion où se confondent richesse et malaise de l'exilé. ${ }^{9}$ Il s'agit pour Ionesco de «traduire» pensées et attitudes issues de deux cultures (nous nous attachons ici à la culture roumaine), de s'adapter et de se réadapter.

Il s'agit aussi de traduire, au sens propre du mot cette fois. Après avoir passé son enfance en France, il arrive au lycée roumain avec une petite pièce de théâtre, «drame patriotique» qu'il avait écrit en français à onze ans et qu'il traduisit en roumain à quatorze ans. Plus tard, de nouveau en France, pendant la guerre, il travaille à Marseille comme traducteur pour les éditions Jean Vigneau. Il traduit, entre autres, l'cuvre posthume d'Urmuz, nom de plume

9. Mais Eugène lonesco est-il l'exilé typique? D'où est-il exilé? Combien de fois s'exila-t-il? Il est né en Roumanie mais quitte le pays de son père pour la France, pays de sa mère, lorsqu'il avait à peine deux ans. À l'âge de quatorze ans, à la suite du divorce de ses parents, il est obligé de revenir, malgré lui, en Roumanie, auprès d'un père qui s'était remarié et qu'il déteste. Il suit les cours d'un des meilleurs lycées roumains et obtient ensuite une licence en français à la Faculté des Lettres de l'Universite de Bucarest. Professeur de français dans un lycée, il se lance dans la carrière littéraire roumaine, écrit des poésies et déploie une activité très intense de critique. En 1938, il part comme boursier à Paris pour une thèse de doctorat sur le thème du péché et de la mort dans la poésie française après Raudelaire, thèse qu'il ne finira jamais. En 1940 il rentre en Roumanie et vit l'angoisse de la guerre. Deux ans après, en 1942, donc à l'âge de 33 ans, il regagne la France pour ne plus revenir dans son pays natal. 
d'un jeune magistrat roumain qui s'était suicidé dans un parc de Bucarest en 1923, œuvre faite «de textes bizarres à l'humour absurde» et aux «paraboles intelligentes» (Non, p. 13). En lui on reconnaît l'un des "grands précurseurs du surréalisme» et une lecture attentive permettrait d'y déceler des influences directes sur l'œuvre de Ionesco. Il s'agit encore pour Ionesco de traduire en français sa première pièce de théâtre la Cantatrice chauve, qu'il avait écrite tout d'abord en roumain au début des années 1940 . A noter, d'ailleurs, que cette version roumaine paraissait beaucoup plus provocatrice, car, comme l'avait fait Tristan Tzara, cet autre écrivain roumain «enfant terrible» du dadaïsme, l'auteur lui-même devait monter sur la scène à la fin de la pièce et lancer des insultes au public: «Bande de salauds, j’aurai votre peau»!

\section{Les Élégies, présentation de la traduction en français de quelques pièces et intérêt pour le théâtre}

Le secret de mon adaptation...? - J'ai changé de désespoir comme de chemise.

Après avoir cherché en vain un pays d'adoption, se rabattre sur la mort, pour, dans ce nouvel exil, s'installer en citoyen.

Emil Cioran

La poésie ne réside pas dans l'expression opulente, mais dans le mot nu.

Eugène Ionesco

Eugène Ionesco groupe ses Élégies en deux cycles: les Élégies pour petits êtres et les Élégies grotesques, structure binaire qu'il allait employer aussi pour l'Hugoliade, mais où le "grotesque» précédera le «tragique». Les élégies comprennent en tout vingtcinq brefs poèmes, d'un style ramassé, dépouillé, voire simple. Néanmoins, l'établissement du texte français a posé de nombreux problèmes délicats. Ayant devant nous des poèmes écrits généralement en vers libres, nous avons pu suivre de très près l'original et donner la version française presque mot à mot. Le premier principe a été de ne jamais supprimer une image ni même un mot de l'auteur; bien au contraire, nous avons préféré procéder par addition pour qu'on comprenne mieux. Dans certains cas où le rythme nous paraît essentiel, le rythme-sens faisant partie du message du poème, nous avons tenté quelques compromis, heureux espérons-le. Nous tenons à signaler que ce fut un plaisir de constater combien proche était parfois le souffle, la respiration poétique de ces deux langues latines. Nous avons essayé de reconstituer le rythme et la rime, lorsque celle-ci existait. Cela a exigé un long travail pendant lequel, fait significatif, la connaissance de l'œuvre dramatique française de Ionesco a considérablement aidé 
à retrouver et respecter autant que possible l'idée de l'auteur. Des rapports invisibles, tels des racines solides, sont apparus sous cette terre féconde de l'œuvre ionescienne.

Or, on voit d'emblée émerger, à partir même des titres des deux cycles des poèmes, une introduction à l'univers du dramaturge et la conception originale de son architecture dramatique. En effet, il y a, dès le début, une constatation d'un grand intérêt sur le caractère novateur de sa dramaturgie. Le dérisoire apparaît sous le double visage du théâtre à venir, ce double masque de la douleur et du rire. L'auteur réunit en un couple insolite le "chant de mort» (l'élégie proprement dite) et la cocasse invention de «l'élégie grotesque». D'entrée de jeu donc, Ionesco lance un genre nouveau, caricatural et dérisoire: «l'élégie joyeuse»!

Cette unique aventure poétique est placée entre deux poèmes écrits à la première personne. Le premier est une Prière touchante par sa naïveté, car elle demande l'impossible pureté. Le dernier, intitulé Souvenir, établit un pont hardi entre le passé et les rives inconnues de l'avenir. Ce diptyque, cette âme ouverte vers le monde, inscrit en larmes et en éclats de rires le jeu du «je»-«moi» multiple, métamorphosé en l'autre, en tous les autres, êtres et objets.

La première parole de l'écrivain est un cri, un appel ardent d'un rayon de lumière dans l'obscurité de la souffrance incomprise. Un être humble, perdu dans. le microcosme des petits êtres - animés ou inanimés -, fait sa Prière:

Un mic soare, Doamne, pentru sufletul meu.

Doamne, eu sunt of frunza, eu sunt o nuca sunt un broscoiu speriat sunt o vrabie ranita.

Mi-au furat toate cuiburile. M'au ajuns toate prastiile.
Un petit soleil, Seigneur, pour mon âme.

Seigneur, je suis une feuille, je suis une noix, je suis une grenouille effrayée je suis un moineau blessé.

On m'a volé tous les nids.

On m'a atteint de toutes les frondes.

Le tout premier sentiment qui se dégage est la peur omniprésente, constante obsédante de toute l'œuvre ionescienne à venir. Mais sous quels visages pitoyables et ridicules, qui nous font presque sourire, non pas de la peur, mais paradoxalement du courage. Oui, du courage de son auto-persiflage, car il se présente comme une "grenouille effrayée», apeurée (en roumain le masculin «broscoi speriat») et comme «un moineau blessé» (en roumain le féminin «vrabie ranita»). Ces petits êtres dérisoires dans leur 
impuissance apparaissent comme de banals petits bavards, mais sont aussi porteurs de riches suggestions symboliques. ${ }^{10}$

Il faut remarquer l'effort du poète pour s'assimiler aux choses et pour s' intégrer à toutes les formes de la création, végétale et animale, et à tous les éléments: air, eau, terre, feu. Naïvement, il demande à être sauvé, et à être repris dans le paradis perdu de l'innocence:

Doamne mic, ridica-ma, si fa-ma fericit

ca pe boii cu coarne nevinovate, ca pe câinii cu ochii de ingeri, ca pe nenuferi, ca pietrele prietenele.
Dieu petit, relève-moi, et rends-moi heureux comme les bœufs aux comes innocentes, comme les chiens aux yeux d'anges, comme les nénuphars, comme les pierres, les amies.

Cette attitude s'oriente non seulement vers un certain panthéisme, mais vers un mutisme causé par la douleur incomprise. Le Solitaire du roman de plus tard, devenu le Personnage lorsque Ionesco transformera le roman en la pièce Ce formidable bordel, sera justement atteint d'une sorte d'aphasie, assistant muet à l'agitation futile du monde. On comprend mieux alors son regard sur ces «grenouilles apeurées", ses semblables, regard s'efforçant de se distancer et d'imiter l'indifférence des "pierres, les amies». ${ }^{11}$

Aux images symboliques et révélatrices des structures de la dramaturgie future, ajoutons aussi l'image des «nénuphars», chargés eux aussi de suggestions, témoins vivants et silencieux, fleurs immuables, flottant sur l'eau stagnante des étangs. Toutes les images suggestives de cette poésie, y compris l'image «des bœufs aux cornes innocentes" annoncent et éclairent l'intrigue principale et le déroulement du conte la Vase. Ionesco transformera plus tard ce conte en scénario pour un film où il jouera lui-même le rôle unique.

Une dernière remarque s'impose sur ce premier poème, car il s'agit d'une prière, «d'un acte d'humilité» venu du cœur. Pour

10. Selon J. E. Cirlot: «The image of the frog represents the transition from the Element of earth to that of water and vice versa... The frog was one of the principul beings associated with the idea of creation and resurrection, not only because it was amphibious but because of its alternating periods of appearance and disappearance (...) The folklore knows of the transformation of prince into frog and vice versa.) (A Dictionary of Symbols, Second Ed., New York, Philosophical Library, 1971, pp. 114-115). Dans cet univers de "petits êtres» le moineau, comme tout oiseau, pourrait symboliser l'âme humaine.

11. Limage de la pierre, «l'amien, semble n'avoir jamais quitté l'auteur qui écrit, en juillet 1986, dans son journal intime: «...et si on en croit Leibniz, qui disait que la pierre elle-même est une pensće qui dort?» (La Quête intermittente, p. 31). 
Ionesco, comme pour Bergson, la prière pourrait être indifférente à son expression verbale, l'élévation de l'âme pouvant se passer de la parole. En effet, tant par sa construction que par son choix de mots et d'expressions, ce poème est d'une simplicité touchante et d'une émotion profonde et vraie qui n'a que faire de toute parure. Des paroles de tous les jours chuchotées dans la solitude, la tête baissée, mais qui ont la valeur d'un cri de supplication qui s'élève vers le ciel. Découverte capitale donc du rôle de la prière chez cet écrivain, appelé trop souvent, contre sa volonté, «dramaturge de l'absurde». L'auteur affirme lui-même dans son dernier volume de mémoires la Quête intermittente (Gallimard, 1987):

La littétature, oui, oui, mord sur la prière, sur la méditation ou sur le recueillement, sur les autres idées qui me viennent, peut-être bénéfiques, peut-être inspirées. Qui sont peut-être des petits, petits, petits pas vers la lumière, vers une illumination. (p. 153)

D'ailleurs, dans ce journal récent Ionesco introduit des paroles en roumain d'une des plus belles prières chantées le jour de Pâques, le jour de la résurrection du Christ:

Avoir la force de vivre la mort, pour ne pas mourir: cu moartea pre moarte calcand (par la mort anéantissant la mort: notre traduction). Naïvement, je dis que je voudrais être sauvé,... que je voudrais tant, comme le voulait et le disait Péguy, faire monter au ciel. (Quête, p. 15)

Il avoue plus loin, revenant toujours à la langue roumaine, langue de cette première cuvre, de cette prière jaillie du fond de son âme, qu' "au jour le jour, de azi pe mâine (je parle aux hommes)... Les mots ne sont pas la parole. Je leur parle... moi qui voulais dire une parole au ciel, la chanter». (p. 35)

Il faut citer, enfin, une affirmation importante sur le rôle de la prière dans son ouvre, affirmation qui s'inscrit dans cette dernière œuvre avec la même profonde simplicité que son premier poème:

Depuis quelques jours je n'ai plus écrit; c'est-à-dire je n'ai plus prié. Écrire, c'est donc bien ma façon de prier. Est-elle bonne? Mauvaise? Efficace? (Quête, p. 151)

Dès le poème qui suit cette Prière, pièce d'ouverture, Ionesco introduit le thème principal de toute son ouvre: le thème de la Mort. Ainsi la petite élégie qui suit, poème de huit courts vers, nous plonge-t-elle dans une atmosphère vague de deuil dans un jardin fleuri. Est-ce la mort d'un petit enfant (Ionesco enfant avait perdu un petit frère)? Les poèmes qui suivent pourraient bien le suggérer. Des rimes s'entendent, harmonie dans ce jardin au par- 
fum «frais et souriant». Voici les versions roumaine et française de la deuxième partie de cette poésie intitulée Élégie:

Ca sa fie linistit

frate blând l'a învelit.

Si acum, cuminte-nchis

deapana albastru vis.
Pour qu'il reste en paix

frère gentil l'a enveloppé.

Et maintenant sage-clos (entre les murs)

déroule son rêve d'azur.

Le texte entre parenthèses dans la traduction correspond à une addition. Nous avons opté pour cette solution afin de conserver la rime. ${ }^{12}$

Dans le poème suivant, intitulé Chanson, le poète s'interroge sur le vol de l'âme innocente de l'enfant:

Alb acum el sade sus

Blanc il reste au-dessus

in gradina, la Isus? dans le jardin, chez Jésus?

Le jardin fleurit s'anime, ainsi que les objets de la chambre de l'enfant plongés dans l'obscurité de la nuit, dans les deux poèmes suivants, intitulés tous les deux Animisme.

$O$, când le sunt frate,

clipele cu lucrurile!

Covorul cu schimonoseli

zâmbeste...

Jos, prin crapaturi

se arata niste guri
0 , lorsque frères, les instants le sont aux choses!

Le tapis sourit aux grimaces...

Là, à travers des trous

des bouches font la moue

En roumain «jos», au début du dernier distique, veut dire «par terre», que nous avons traduit par un monosyllabe: «là». La traduction littérale du vers suivant est «se montrent des bouches». Nous avons employé l'expression «faire la moue» non seulement à cause de la rime, mais parce qu'elle nous semble trouver sa place parfaitement à côté de l'expression employée par l'auteur, «le tapis sourit aux grimaces"».

On connaît la fortune des procédés similaires dans tout le théâtre de Ionesco, où les champignons envahissent la scène et le cadavre grandit et s'envole emportant avec lui Amédée (Amédée ou Comment s'en débarrasser) ou bien les murs du palais du roi qui s'écroulent dans le Roi se meurt. Où est la frontière entre le

12. Le but du présent essai n'est pas essentiellement de présenter notre traduction en en justifiant les choix, les Élégies étant encore inédites en traduction; aussi ne nous étendrons-nous pas sur notre lecture-traduction. Nous nous proposons de combler cette lacune lorsque les Élégies seront publiées. Notons cependant que l'image des umurs» qui n'apparaît pas explicitement dans cette élégie correspond exactement à l'idée exprimée par ces vers: elle sera reprise et explicitée en ces termes mêmes, plus tard, dans les mémoires et le théâtre. 
réel et l'onirique? Son théâtre nous paraît bien avoir son origine dans ces premières images que l'on découvre dans les poésies.

Le même thème de la Mort est traité dans la poésie Ballade. Le poète imagine une visite nocturne, invisible, d'un personnage mystérieux, de la mort elle-même. En voici un fragment:

$\begin{array}{lll}\text { (1) } \begin{array}{l}\text { Peste ape trece } \\ \text { nimeni n'o petrece. }\end{array} & \begin{array}{l}\text { Elle passe au-dessus des puits } \\ \text { personne ne la conduit. }\end{array} \\ \text { (2) } \begin{array}{l}\text { Poposeste'n sat } \\ \text { si nici un latrat. }\end{array} & \begin{array}{l}\text { Arrive chez les villageois } \\ \text { et pas un chien n'aboie. }\end{array} \\ \text { (3) } \quad \begin{array}{l}\text { Soseste'n ograda } \\ \text { cu pasi de zapada. }\end{array} & \begin{array}{l}\text { A pas de neige s'arrête } \\ \text { dans la cour muette. }\end{array} \\ \text { (4) } \begin{array}{l}\text { Puneti dese perdele } \\ \text { sa nu vada n'ele. }\end{array} & \begin{array}{l}\text { Mettez des rideaux épais } \\ \text { pour qu'elle n'y voie mais. }\end{array} \\ \text { (5) } \quad \begin{array}{l}\text { Puneti lacat la poarta } \\ \text { sa nu il sparga. }\end{array} & \begin{array}{l}\text { A la porte mettez lourd cadenas } \\ \text { qu'elle ne le casse pas. }\end{array} \\ \text { (6) Noi sa nu tipam } & \begin{array}{l}\text { Tenons-nous coi, } \\ \text { sa nu ne miscam. }\end{array} & \text { pas le son d'une voix. }\end{array}$

La traduction littérale du premier distique est «Sur les eaux passe». Nous avons traduit "eaux» par «puits». Remarquons aussi le verbe «passe», qui en roumain, à la forme de la troisième personne du singulier, ne se confond pas avec l'impératif grâce à une désinence différente. Au dernier vers du quatrième distique, à la place de la négation habituelle «pas» nous avons eu recours à une négation archaïque «mais», ce qui a permis de préserver et le rythme et la rime. Pour les mêmes raisons, les vers du troisième et du dernier distiques cités ont été inversés. Même si l'emploi de «mais» au sens de «plus» n'est plus usité, il est si proche du roumain «maï» (plus) que nous n'avons pu y résister.

Le mystère est renforcé par l'absence totale du sujet de tous les verbes à la troisième personne du singulier. C'est un procédé grammatical tout à fait possible en roumain, mais dans ce cas il laisserait dans une obscurité complète l'identité du sujet. Il n'y a qu'une référence (et de la manière la plus discrète possible) au visiteur inconnu et invisible, référence qui apparaît au début, au quatrième vers sous la forme du pronom objet direct féminin, le plus court de la langue, le pronom «o». Voyelle qui par son timbre grave et sombre sert à rendre un bruit sourd. Elle est à la fois la voyelle de la surprise, de l'angoisse et de la douleur. Plus encore, ce pronom est l'objet direct dans une phrase dont le sujet est «nimeni», c'est-à-dire "personne», pronom indéfini suivi de la négation ne ( $\mathrm{n}$ '). Précédé de deux négations et de l'indéfini, le sombre écho de «o» enveloppe de mystère la série de verbes ellyptiques qui suit. Évidemment, ce mystère au niveau de la 
structure grammaticale ne peut pas être fidèlement rendu dans la version française. Nous avons été obligée d'introduire le pronom personnel sujet «elle», absent en roumain.

Nous avons tenté de rendre autant que possible la simplicité des rimes suivies du roumain et, dans cet effort, nous avons opté non pas pour une réduction du vers (nous l'avons conservé presque mot pour mot) mais pour une addition de deux adjectifs qui ne figurent pas dans les vers roumains: «muette» (pour «cour») et «lourd» (pour «cadenas»).

A ce propos, il faut faire ici une remarque essentielle pour l'esthétique poétique de Ionesco: l'adjectif y est fort rare. Le seul adjectif qualificatif choisi par l'auteur dans ce poème est «épais» pour «tideaux». L'absence de l'adjectif qualificatif est une réaction parfaitement saine contre les abus dont il a horreur chez les romantiques ou même chez les symbolistes.

Poète, Ionesco aime les formes brèves, les phrases extrêmement courtes sur le modèle: sujet, verbe, complément. Il n'y a ni effusion de sentiments (bien qu'il s'agisse d'une élégie), ni mots savants, ni vers aux tournures inattendues. Et cependant, malgré l'extrême économie de moyens, on sent le suspens et le frisson de l'angoisse diffuse. Mais par quel procédé? Par une mise en scène. Le sujet, protagoniste invisible et muet, s'insinue subrepticement au crépuscule, bandit terrorisant les gens, envahissant le monde. C'est le Mal, c'est la Mort, c'est exactement la mise en scène de la pièce de théâtre, le Tueur sanis gages, pièce qui fut développée à partir de la nouvelle la Photo du Colonel.

Le thème de la mort est comme un fil touge qui traverse toute la création ionescienne. Thème classique s'il en est, il apparaît dans ce poème avec toute la puissance de l'originalité que lui confère Ionesco. Poème, nouvelle, pièce de théâtre, mémoires, longue quête inconsolable, effort infatigable pour dompter l'indomptable! Devant son destin l'homme apparaît comme un pantin, comme une marionnette ridicule.

Le thème de la mort recoupe un deuxième thème, celui du dérisoire de la condition humaine. Une série de plusieurs élégies, à notre avis les moins réussies, introduisent des poupées de cire, de porcelaine ou de chiffon remplies de son. Mortes, elles sont conduites au cimetière par des chevaux de bois, des soldats de plomb, des pierrots et des arlequins «en tenue bizarre» qui forment un cortège funèbre derrière «le cercueil de carton», sur «un chemin de papier", pendant que «le pauvre pierrot perd le son par ses coudes déchirés»...

Ces personnages insolites, poupées et marionnettes pitoyables et ridicules, automates sans âme, font sans doute penser aux 
personnages de la Cantatrice chauve de plus tard. ${ }^{13}$ Sur le plan de l'existence, ils suggèrent la farce, la bouffonnerie et l'absurdité de tout devant la mort irréversible. A cet égard, ces poèmes introduisent le thème des grandes pièces à venir du Tueur sans gages, de Jeux de massacre ou de Macbett. Le jeune Ionesco de l'époque des poèmes roumains écrivait d'ailleurs dans ses essais de critique littéraire:

Nous sommes de pauvres gosses, abandonnés dans le monde, dans cette immense bâtisse en démolition. Au bord de l'abîme nous jouons à la poupée, à ainsi font font font les petites marionnettes. (Non, p. 86)

Dans ce contexte les larmes pourraient nous sembler inutiles, mais chez Ionesco les deux attitudes diamétralement opposées ont toujours coexisté. Car les larmes sont vraies dans une autre petite Élégie:

Prietene, sa plâgem:

o lacrima va fi pentru

frunza galbena o lacrima

pentru trandafirul scuturat,

o lacrima pentru fata moarta,

o lacrima pentru durerea

fiecarui om.

O lacrima pentru fiecare

piatra

pentru fiecare pom

pentru fiecare stea

si pentru Ideal.
Ami, pleurons:

une larme pour la feuille jaunie

une larme pour la tose qui a perdu

ses pétales,

une larme pour la jeune fille

morte,

une larme pour la douleur de

chaque homme.

Une larme pour chaque

pierre

pour chaque arbre

pour chaque étoile

et pour l'Idéal.

Si l'auteur a toujours condamné catégoriquement le ton pathétique, il avoue dans sa toute dernière œuvre que c'est justement parce qu'il a gardé cette nature juvénile «vulnérable» qu'il a pu se débattre pour "des idées, des idéaux»:

Tant que je serai fragile, sensible... ce seront des larmes d'un jeune, qui, de mes yeux, couleront, les larmes d'un jeune, d'un enfant, d'un adolescent qui, par je ne sais quel souvenir

13. Ulıe étude que nous allons faire paraître bientôt démontrera à quel point le monologue des grandes pièces de théâtre de Ionesco est l'héritier direct des Élégies de jeunesse. De même, il faut remarquer que ce n'est pas seulement dans la Cantatrice chauve que ces personnages-poupées apparaissent mais dans toute sa dramaturgie. Dans Victimes du devoir, Choubert développe un long monologue poétique et s'adresse à sa femme, Madeleine "petite vieille, poupée vieille... Notre jeunesse perdue, les larmes deviennent des sources pures... des sources de vie, des sources immortelles... Les fleurs fleurissent-elles dans la boue...» (Théâtre l, Gallimard, p. 197). 
d'un paradis perdu, trouva le malheur, le désastre de ce monde, inacceptable. (Quête p. 25)

Est-ce le souvenir de sa poésie de jeunesse qui l'entraîne à s'exprimer par cette inversion poétique du complément «des larmes d'un jeune, qui, de mes yeux, couleront, les larmes d'un jeune...»?

La portée du choc des personnages de la Cantatrice chauve, pauvres automates déréglés, aurait peut-être été mieux comprise et mise en valeur si, à l'époque, on avait connu ces poèmes roumains. En effet, l'homme-pantin ou poupée-marionnette, l'homme «né trompé», l'homme angoissé élève une "prière muette» dans un Pays de carton et d'ouate, tel qu'il apparaît dans le poème du même titre et que l'on peut considérer comme un des plus beaux du recueil. En voici un fragment:

Dans ce pays-là on ne distingue pas la pierre

de l'oiseau ou de l'âme:

Ils sont d'ouate et de carton.

Qui le veut peut sortir son âme,

la pose à côté

et la regarde comme un être étranger:

j'ai vu des âmes d'arbres, d'oiseaux, d'hommes.

Les hommes-poupées chantent une prière muette:

Leur Dieu a une barbe blanche.

Hommes-poupées et âmes d'ouate!

Sourires de pâte!

Arbres de caoutchouc!

Yeux candides et fixes!

Les couleurs sont pâles, ne crient pas.

L'espace a deux mètres cubes.

Le feu est un torchon rouge que l'on prend dans la main.

Une atmosphère insolite se dégage de ce poème, atmosphère caractéristique de la dramaturgie ionescienne. Ce n'est pas uniquement la caricature du quotidien, du banal, son grossissement, c'est surtout la projection du banal métamorphosé dans un monde insolite. Le côté tragique du banal rendu ainsi irréel nous apparaît beaucoup plus douloureux et, paradoxalement, plus comique à la fois. Voici, à notre avis, la phrase-clé du recueil: «Qui le veut peut sortir son âme/ la pose à côté/ et la regarde comme un être étranger»... Paraphrasons et nous reconnaissons une pièce de théâtre célèbre de l'auteur: Qui le veut peut devenir Rhinocéros et regarder ses cornes dans le miroir! Tout le monde le fait! Dans $C e$ formidable bordel (pièce de la maturité) qu'est le monde, n'est devenu banal que ce qui n'aurait jamais dû le devenir! 
La société s'empare de l'individu et le vide de son âme, le transformant en automate. Cette constatation nous conduit au troisième thème que nous avons identifié dans les Élégies, celui de la déshumanisation de l'être social ou encore de la Mort-dansla-vie.

Un «je-autre» constate sa dissolution sous les coups implacables du Mal, sans pouvoir se défendre. Comme dans un cauchemar il assiste tout simplement à ce spectacle incompréhensible. C'est le thème du beau poème l'Individu fatigué:

$\begin{array}{ll}\text { Cineva rupea din el } & \begin{array}{l}\text { Quelqu'un arrachait } \\ \text { (des morceaux de son être) } \\ \text { petit à petit. }\end{array} \\ \text { câte putinel. } & \text { Il fut laissé décoloré } \\ \begin{array}{l}\text { La lasat decolorat } \\ \text { si cutremurat. }\end{array} & \text { et s'est mis à trembler. } \\ \text { Mirat s'a pipait } & \text { Étonné il s'est palpé } \\ \text { dar nu s'a regasit. } & \text { mais il ne s'est plus retrouvé. } \\ \text { Din el o forta rea } & \text { De lui une force méchante } \\ \text { crestea si-l cuprindea. } & \text { grandissait et l'enveloppait. } \\ \text { Obositul ins } & \text { L'individu fatigué } \\ \text { s'a lasat cuprins. } & \text { s'est laissé posséder. }\end{array}$

Le premier vers nous a donné du fil à retordre, car en roumain le verbe «arracher», ici «rompre», n'a pas besoin d'objet direct. Selon la traduction littérale le vers affirme tout simplement: "Quelqu'un rompait de lui», c'est-à-dire arrachait, enlevait des petits morceaux comme on rompt et on mange des petits morceaux de pain. Comment rendre fidèlement cette image sans ajouter le complément direct en français? Plus tard, dans la pièce de théâtre Victimes du devoir, Ionesco traduira cette image dans le pénible et douloureux leitmotif du devoir: «Mange! Avale! Mastique!...»

Pour le reste du poème, la série des verbes au passé composé et à l'imparfait correspond exactement dans les deux langues. Remarquons qu'il y a mêtme une sorte d'harmonie entre les terminaisons des imparfaits "ea» et «ait», harmonie vocalique qui se prolonge, suggérant l'idée de continuité.

$\mathrm{Si}$, selon Ionesco, l'histoire de l'humanité demeure un cauchemar dont nous cherchons à nous éveiller, il a réussi parfaitement à donner ce sentiment dès cette première œuvre, ces poèmes de jeunesse. On y voit émerger les procédés de son théâtre onirique, de ses nouvelles, telle la Vase, histoire racontée à la première personne par ce «je-autre» qui assiste à sa propre décomposition, à sa propre mort (et «vit» pour la raconter!). 
Pour conclure cette étude, quelques remarques d'ensemble s'imposent sur le style de la création poétique de lonesco. Il a toujours été le meilleur critique de son propre théâtre. Il l'est également pour sa poésie, mais indirectement seulement. En effet, dans Hugoliade, en définissant la poésie, il définit aussi le style de ses Élégies:

La poésie n'est ni vocabulaire, ni grammaire... ni expression lexicale, mais mode d'expression. Elle est une émotion dite et non pas spéculée. Elle est cri et non discours. Elle n'est même pas le développement d'une exclamation, comme le dit Valéry, mais l'exclamation même... La poésie participe à la vie la plus pure et la plus élémentaire de l'esprit. (Hugoliade, p. 26)

Sa poésie s'est donc définie en réaction contre la poésie de Victor Hugo et, en même temps, en réaction contre la poésie roumaine contemporaine. En critiquant le poète roumain Arghezi, il invite avec ironie à enlever les mots rares, les trouvailles ou les belles métaphores, les adjectifs et les noms abstraits, les innovations syntaxiques, les discours et la thétorique, les symboles suggestifs ou l'expressivité sonore. Que tout cela se diffuse aux quatre vents! Et il ajoute:

Mais en se diffusant, ce matériel inorganisé, sans ossature, s'est dissout, et la poésie s'est volatilisée. On ne saurait trouver... ni colonne vertébrale, ni vigueur intérieure, mais seulement la chair flasque de mots désintégrés... Après la désintégration, c'est-à-dire la diffusion dans le langage des éléments extérieurs à la poésie, il subsiste néanmoins, chez les grands poètes, quelque chose d'indestructible: l'architecture lyrique... L'essence de l'art n'est autre que la particularité, l'unicité de l'intuition lyrique. C'est pourquoi il existe de grands poètes ayant un vocabulaire réduit, voire pauvre Racine ou Rilke par exemple. (Non, pp. 63-64)

Retenons surtout l'expression architecture lyrique, car plus tard l'auteur caractérisera également son théâtre d'architecture. Tous ses poèmes ne représentent ni le «discours sentimental ou thétorique», qu'il déteste chez les poètes romantiques, ni la «composition morcelée, véritable mosaïque et mécanique», qu'il déteste chez les poètes symbolistes, mais la "vérité de l'émotion», vérité de «la mise en forme de figures émotives», une "construction» (Non, pp. 30-32), ou, en d'autres termes, une mise en scène. Sur quoi le jeunc poète débouche-t-il ainsi? Sur le théâtre. L'expérience poétique le conduit à la dramaturgie. Il l'affirme d'ailleurs directement à l'ćpoque, sans savoir tout ce que cette affirmation aura de prophétique pour sa propre carrière d'écrivain. Après avoir écrit de la poésie, après avoir tenté les limites des mots, il ne garde que 
pour le théâtre - pour l'acte vivant de la mise en scène - la capacité de transmettre le lyrisme pur, le tragique intérieur. Ce n'est qu' «au théâtre» que ce «tragique intérieur peut ne pas se banaliser» et arriver à un profond «repliement sur soi», affirme-t-il dans un essai de Non (p. 45).

Il ne fait aucun doute que dans les Élégies pour petits êtres le langage est dépouillé jusqu'à «l'ossature». Comme nous l'avons constaté, certains poèmes deviennent tout simplement des mises en scène de l'insolite. Les poèmes introduisent les thèmes obsessifs qui traverseront son ouvre d'un bout à l'autre: la hantise de la mort, l'oscillation entre l'espoir et la détresse, l'étonnement qui remplit une âme cherchant «sa grâce» et questionnant l'inconnu, le vent, le rêve, le vide, le néant, la révolte muette et douloureuse contre le sort de l'homme pantin, caricature pathétique de luimême, le grotesque de la farce qui suscite le rire aux dépens de tous, y compris de l'auteur.

Il ne fait aucun doute non plus que l'architecture du volume de poèmes basée sur l'opposition entre des «élégies», chants de deuil, et des «élégies grotesques»(!) annonce également la structure dynamique de la dramaturgie. Les limites de cet essai ont seulement permis d'aborder le premier cycle. Il restera à montrer, dans une deuxième étude sur les Élégies de Ionesco que le cycle des «élégies grotesques» mérite également d'être connu, car par ces petites pièces bouffonnes l'auteur pourrait être encore plus près du théâtre.

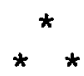

Il n'est certes pas indifférent que nous ayons choisi de présenter quelques-unes des pièces des Élégies traduites en français par nos soins pour situer le théâtre de Ionesco dans une perspective qui nous paraît plus juste. Les Élégies roumaines, cuvres de jeunesse à ce jour intraduites, sont pour ainsi dire la preuve $a$ contrario et paradoxale du caractère central de la traduction dans l'univers mental de l'auteur de la Cantatrice chauve. Il suffit d'être confronté à ces pièces encore inconnues en français pour le voir, tant elles sont significatives d'une cuvre qui s'épanouira dans cette langue. Comme nous pensons l'avoir montré, elles jettent une lumière nouvelle sur le théâtre. 


\section{Références}

\section{A. Euvres d'Eugène Ionesco}

IoNESCO, Eugène (1931). Elegii pentru fiinte mici, Craïova, Editura "Cercul Analelor Române».

(1934). $N u$. Texte français: Non (1986) traduit du roumain et annoté par Marie-France Ionesco. Paris, Gallimard.

(1987). La Quête intermittente, Paris, Gallimard.

(1954). Théâtre, Tome I, Paris, Gallimard.

(1958). Théâtre, Tome II, Paris, Gallimard.

(1963). Théâtre, Tome III, Paris, Gallimard.

(1966). Théâtre, Tome IV, Paris, Gallimard.

(1971). Théâtre, Tome V, Paris, Gallimard.

(1975). Théâtre, Tome VI, Paris, Gallimard.

(1981). Théâtre, Tome VII, Paris, Gallimard.

(1973). Le Solitaire, roman. Paris, Mercure de France.

B. Euvres sur la littérature roumaine

Dictionarul literaturii române (1979). Bucuresti, Academia Româna, Institutul de Linguistica si Istorie Literarà a Universitàtii Al. I. Cuza. Iasi.

Calinesco, Georges (1982). Istoria Literaturii Române de la origni pâna în prezent. Bucuresti, Editura Minerva, 948 pages inquarto.

Croculescu, Serban, Alex. Piru et coll. (1973). Istoria Literaturii Române (IV Volumes). Bucuresti, Editura Academiei.

JuIN, Hubstr (1961). Introduction à la poésie roumaine. Paris, Collection Messidor.

Munteanu, George (1980). Istoria Literaturii Române. Epoca Marilor Clasici. Bucuresti, Editura Didactica si Pedagogica. 